\title{
RADIOTERAPIA TRAS PROSTATECTOMIAA RADICAL: FACTORES DESCRIPTIVOS, EVOLUTIVOS Y DE PRONÓSTICO EN UNA PRÁCTICA ASISTENCIAL EMERGENTE.
}

\author{
Mำ Victoria de Torres Olombrada, Carmen González San Segundo, Juan A. Santos Miranda, \\ Carmen Ibáñez Villoslada, Juan I. Martínez Salamanca', Felipe Herranz Amo' y Felipe A. Calvo \\ Manuel.
}

Servicio de Oncología Radioterápica y Servicio de Urología'. Hospital General Universitario Gregorio Marañón. Madrid. España.

\begin{abstract}
Resumen.- OBJETIVO: Recientemente se han comunicado firmes evidencias por la EORTC (European Organisation for Research and Treatment of Cancer - ensayo 229111 y el SWOG (Southwest Oncology Group - ensayo 8794), señalando que la radioterapia (RT) es un tratamiento eficaz tras la prostatectomía en pacientes con alto riesgo de fracaso bioquímico. Definir el momento óptimo para su administración e identificar factores de riesgo predictivos de recidiva, son objetivos transcendentes para guiar la práctica asistencial.

MÉTODOS: Desde 1993 hasta 2003, 597 pacientes fueron tratados con prostatectomía radical en el Hospital General Universitario Gregorio Marañón y áreas de referencia asistencial. 166 pacientes (28\%) desarrollaron una recidiva bioquímica (definida como PSA $\geq 0,5$ $\mathrm{ng} / \mathrm{ml}$ e incluyendo aquellos casos con persistencia tumorall. Cuarenta y dos, recibieron tratamiento con RT
\end{abstract}

$M^{a}$ Victoria de Torres Olombrada Servicio de Oncología Radioterápica Hospital General Universitario Gregorio Marañón Doctor Esquerdo $\mathrm{n}^{\circ} 46$ 28007 Madrid. (España). marividetorres@yahoo.es Trabajo recibido: 10 de enero 2007.
(78\% tras fallo bioquímico). La dosis media de RT fue de 66 Gy [60-74].

RESULTADOS: Variables clínicas: Edad media: 68 años [49-80], media del PSA al diagnóstico: $29,8 \mathrm{ng} / \mathrm{ml}$ [2,6-475], Gleason prequirúrgico $\geq 7$ : 65\%. Variables patológicas: Tras la prostatectomía, los pacientes tenían datos de mayor agresividad histológica que la definida previamente en las biopsias, apareciendo Gleason $\geq 7$ en el $81 \%$ de los pacientes. El 83\% a su vez tenían borde afecto y en el $90 \%$ de los casos el estadio era pT3pT4. Variables evolutivas: El tiempo medio de aparición de la recidiva bioquímica fue de 22,2 meses, con un intervalo de 10,5 meses desde el diagnóstico hasta el inicio de la RT. La SG fue de $86 \pm 6 \%$ a los 5 años y la Supervivencia Libre de Fracaso Bioquímico (SLFB) fue de $76 \pm 4 \%$ a los 5 años.

Los factores predisponentes para la recidiva fueron: $P S A>2 \mathrm{ng} / \mathrm{ml}$ al inicio de la RT ( $p=0,03)$, persistencia tumoral $(p=0,05)$ y Gleason $\geq 7$ tras la prostatectomía $(p=0,01)$. No se observó un aumento de la toxicidad grado 3 y 4 en los pacientes tratados con RT.

CONCLUSIONES: La RT tras prostatectomía es un tratamiento eficaz de rescate tras recidiva bioquímica o persistencia cuando el PSA no supera los $2 \mathrm{ng} / \mathrm{ml}$. En nuestra serie, el Gleason $\geq 7$ es un factor adverso de respuesta a la RT de rescate. No existe un aumento de la toxicidad severa. La mejora de las técnicas de estadificación prequirúrgica, el papel de la hormonoterapia adyuvante y la selección de los pacientes para RT adyuvante centran los estudios actuales de los tratamientos tras prostatectomía.

Palabras clave: Prostatectomía. Radioterapia.

Recidiva bioquímica. 
Summary.- OBJECTIVES: Recently it has been reported in the EORTC (European Organisation for Research and Treatment of Cancer) trial 22911 and the SWOG (Southwest Oncology Group) 8794, the evidence that radiotherapy (RT) is an effective treatment after the prostatectomy in patients with high risk of biochemical failure. We analyze predictor factors of biochemical relapse and the potential benefits induced by rescue treatment are the main purposes of our study.

METHODS: From 1993 to 2003, 597 prostatectomy were followed at Hospital Universitario Gregorio Marañón de Madrid, identifying 166 patients (p) (28\%) of biochemical failure (defined as PSA $\geq 0^{\prime} 5 \mathrm{ng} / \mathrm{ml}$, including post-surgical persistent values). $42 p$ received $R T$ (78\% due to delayed PSA relapse). The median total dose was 66 Gy [60-74].

RESULTS: Clinical variables: Median age: 68 years [49-80], median PSA at diagnosis: $29,8 \mathrm{ng} / \mathrm{ml}[2,6-$ 475]; presurgical Gleason $\geq 7$ : 65\%. Histological variables: Prostatectomy induces stage migration to superior T (pT3-T4: 95\%) and Gleason categories (27: $81 \%$ ). $83 \%$ of relapsed $p$ had positive margins and $90 \%$ had pT3-pT4. Outcome variables: median time to biochemical recurrence was 22,2 months. Median time interval between biochemical failure and RT was 10,5 months. Overall survival (5 years) was $86 \pm 6 \%$. Freedom-frombiochemical failure at 5 years was $76 \pm 4 \%$.

RT had poor survival in $p$ with PSA $>2 \mathrm{ng} / \mathrm{ml}$ preRT ( $p=0,03)$, post-prostatectomy persistant disease $(p=0,05)$ and Gleason score $\geq 7(p=0,01)$. No increased grade 3-4 uro-rectal toxicity was observed.

CONCLUSIONS: RT after prostatectomy improves freedom-from-biochemical failure in $p$ with PSA values below $2 \mathrm{ng} / \mathrm{ml}$. In our experience, Gleason score $\geq 7$ is a negative predictor of response. There is no severe toxicity in our series. Improvement of the staging presurgery, the role of the adjuvant androgen deprivation and selection of patients for adjuvant RT focus current studies on treatment after prostatectomy.

Keywords: Prostatectomy. Radiotherapy.

Biochemical failure.

\section{INTRODUCCIÓN}

El cáncer de próstata se sitúa a la cabeza en la incidencia de los tumores malignos sólidos en el varón en EEUU (1) y es la segunda causa de muerte por cáncer en los varones en España (2). Se estima que el $85 \%$ de los casos están localizados al diagnóstico. La prostatectomía se ha considerado el tratamiento estándar en esta situación. Sin embargo, el $40 \%$ de los pacientes tratados mediante cirugía radical desarrollarán una progresión bioquímica en el curso de su evolución (3-7).

La indicación de la radioterapia (RT) tras la prostatectomía es controvertida. Mientras que su empleo ha demostrado un mejor control local y de la supervivencia libre de fracaso bioquímica (SLFB), el beneficio en la supervivencia global no ha sido confirmado (8-9).

Definir la indicación y el momento más adecuado para la administración de la RT (adyuvante o de rescate tras la elevación del PSA) e identificar los factores de riesgo predictivos de recidiva, tras la prostatectomía, son objetivos de interés para guiar la futura práctica asistencial, tras analizar la experiencia institucional del Hospital General Universitario Gregorio Marañón (HGUGM) en los últimos 10 años.

\section{MATERIALES Y MÉTODOS}

Desde 1993 hasta 2003, un total de 597 pacientes fueron tratados con prostatectomía radical en el HGUGM y sus áreas de referencia asistencial. 166 pacientes recidivaron $(28 \%)$ considerándose como recidiva bioquímica la elevación del $\mathrm{PSA} \geq 0,5$ $\mathrm{ng} / \mathrm{ml}$ a los tres meses de la cirugía (10).

Cuarenta y dos pacientes recibieron radioterapia con diversas indicaciones: 2 de ellos con finalidad adyuvante, 7 por persistencia de PSA elevado tras la cirugía y 33 por recidiva bioquímica en su curso postoperatorio. La estadificación inicial se realizó mediante anamnesis, tacto rectal y determinación del PSA. El estudio de extensión no incluyó gammagrafía ósea ni TAC de forma sistemática, al tratarse de pacientes de bajo riesgo de diseminación sistémica o ganglionar pélvica. En el seguimiento se realizaron las pruebas descritas, con determinaciones de PSA trimestrales al inicio y, posteriormente, cada 6 meses. La radiografía de tórax se realizó con periodicidad anual y se solicitaron otros estudios complementarios sólo cuando la clínica del paciente era sugestiva de enfermedad metastásica.

Tras la cirugía, se consideró margen quirúrgico afecto cuando la tumoración contactaba con los márgenes de resección o infiltraba los mismos. Se analizaron además otras variables histológicas como la presencia de afectación extracapsular, la infiltración perineural o la invasión linfovascular.

A todos los pacientes se les realizó TAC de planificación radioterápica. Desde el año 2000 la 
dosimetría se realizó en tres dimensiones (3D). En la TAC de localización se identificaron y contornearon el PTV (planning target volume), la vejiga, el recto y las cabezas femorales. El PTV incluía el lecho de prostatectomía con margen de seguridad de $1 \mathrm{~cm}$ en todos los pacientes. El contenido pélvico se trató en aquellos pacientes a los que no se les realizó linfadenectomía en el momento de la cirugía inicial y/o a los que presentaban factores de riesgo de afectación ganglionar tras analizar la pieza quirúrgica (Gleason $>7$, pT3 y pT4).

Se registró la toxicidad del la RT según la escala de la EORTC/RTOG (1 1) con una periodicidad semanal durante la irradiación y, posteriormente, en cada revisión semestral.

El tratamiento se realizó con técnica isocéntrica, campos convergentes y fotones de alta energía de un acelerador lineal (6 y $15 \mathrm{MV}$ ). La dosis media fue 66 Gy (60-74). Los puntos de especificación y prescripción de dosis siguieron las recomendaciones de la International Commission on Radiation Units and Measurements (ICRU 62) (12).

Para el análisis estadístico se utilizó el programa SPSS (Statistical Package for the Social Sciences) en su versión 12.0. Los tiempos de vida se midieron en meses. Para valorar el efecto de la RT tras la prostatectomía, la supervivencia libre de fracaso (SLF) se midió desde la fecha de inicio de la RT hasta la aparición de la recidiva bioquímica según los criterios de la American Society for Therapeutic Radiology and Oncology (ASTRO 1996) (3 elevaciones progresivas del PSA), la recidiva locorregional o las metástasis sistémicas. Asimismo, se contabilizó el tiempo de aparición de la recidiva bioquímica desde la prostatectomía y el periodo desde dicha fecha hasta el inicio de la RT. Para la supervivencia global (SG) se consideró el tiempo transcurrido desde la prostatectomía hasta el fallecimiento del paciente o fecha de último control.

\section{RESULTADOS}

La edad media de los pacientes en el momento del inicio de la RT fue de 68 años [49-80]. El $71 \%$ tenían un índice de Karnofsky del 100\% (29\% restante entre el $80-90 \%$ ).

\section{TNM}

Las categorías de estadificación se describen en la Tabla I. Se objetivó una infraestadificación entre el estadio prequirúrgico y tras prostatectomía: sólo tres pacientes mantenían o disminuían el estadio $T$ tras la cirugía. La linfadenectomía se realizó en 37 pacientes y ninguno resultó $\mathrm{pN}+$.

\section{Gleason pre y postquirúrgico}

Previo a la cirugía, se dispone de la clasificación según la escala de Gleason de 38 pacientes de los 42 analizados, de los que el $65 \%$ tenía un grado comprendido entre 7 y 10 (Tabla II).

TABLA 1. CORRELACIÓN EN LA CATEGORIA DE ESTADIFICACIÓN T PRE Y POSTQUIRÚRGICA.

\begin{tabular}{|c|c|c|c|c|c|c|c|}
\hline & pT2a & pT2b & pT2c & pT3a & pT3b & pT4a & TOTAL \\
\hline cTlc & & 1 & & 4 & 4 & 1 & 10 \\
\hline cT2a & & & 1 & 13 & 1 & 1 & 16 \\
\hline cT2b & 1 & & & 2 & & & 3 \\
\hline cT2c & & & & 1 & 5 & 1 & 7 \\
\hline cT3a & & & & & 2 & & 2 \\
\hline cT3b & & 1 & & & 1 & 2 & 4 \\
\hline TOTAL & 1 & 2 & 1 & 20 & 13 & 5 & 42 \\
\hline
\end{tabular}

c: Estadio clínico. p: Estadio patológico. 
Tras la cirugía, el análisis de la pieza quirúrgica reveló un incremento del grado de agresividad en el $81 \%$ de los pacientes, con un aumento del porcentaje de pacientes con Gleason $\geq 7$ que migró del $65 \%$ al $89 \%$.

\section{Borde afecto}

Treinta y cinco pacientes (83\%) presentaban un margen quirúrgico positivo, generalmente en relación con la afectación extracapsular (pT3a) o con la infiltración de las vesículas seminales (pT3b).

\section{PSA}

En la Tabla III se registra la evolución del PSA durante el seguimiento. En 7 pacientes el PSA a los tres meses de la cirugía no llegó a ser indetectable, lo que se interpretó como persistencia local de enfermedad. En este grupo de pacientes la RT se inició a los 4-6 meses (margen de recuperación de la incontinencia urinaria postquirúrgica).

El tiempo medio transcurrido desde la cirugía hasta la primera elevación del PSA en los pacientes con recidiva bioquímica fue de 22,2 meses [3-69].

A los 2 meses de finalizar la RT el PSA medio fue de $3 \mathrm{ng} / \mathrm{ml}$ descendiendo este valor hasta 0,8 $\mathrm{ng} / \mathrm{ml}$ en el nadir.

\section{Indicación de la radioterapia}

En 33 pacientes la indicación de la RT fue por recidiva bioquímica (78\%). En un 16\% de los casos se indicó el tratamiento por persistencia de un PSA elevado después de la cirugía y tan sólo un $4,8 \%$ se consideró como tratamiento adyuvante programado. La mediana de tiempo transcurrida desde el diagnóstico de recidiva bioquímica hasta el inicio de la RT fue de 10,5 meses [2-58]. Al inicio de la RT el PSA medio fue de $4,1 \mathrm{ng} / \mathrm{ml}$ [0-19]. En 20 pacientes se documentó histológicamente la recidiva en el lecho quirúrgico.

\section{Técnica de radioterapia}

La pelvis se incluyó dentro del volumen de tratamiento en el $71 \%$ de los pacientes. Se empleó una técnica de cuatro campos convergentes, conformados, hasta alcanzar una dosis total acumulativa de $45 \mathrm{~Gy}$, en fraccionamiento convencional.

La dosis media que se administró en el lecho de prostatectomía fue de 66 Gy [60-74], con idéntico fraccionamiento, y utilizando $4-6$ campos conformados.

Un paciente suspendió el tratamiento a los 18 Gy por reagudización de una enfermedad autoinmune.

\section{Toxicidad}

Toxicidad genitourinaria. El $73 \%$ de los pacientes no comunicó síntomas durante la RT registrables como eventos tóxicos, el 16\% refirió molestias de grado 1, y los restantes una toxicidad grado 2 .

TABLA II. MIGRACIÓN EN LOS VALORES DEL ÍNDICE ACUMULADO DE GLEASON PRE Y POSTQUIRÚRGICOS.

\begin{tabular}{|c|c|c|c|c|c|c|}
\hline \multirow{2}{*}{ GLEASON INICIAL } & \multicolumn{7}{|c|}{ GLEASON POSTQUIRÚRGICO } & \multirow{2}{*}{ TOTAL } \\
\cline { 2 - 7 } & 5 & 6 & 7 & 8 & 9 & 1 \\
\hline $\mathbf{3}$ & 1 & 0 & 0 & 0 & 0 & 3 \\
\hline $\mathbf{4}$ & 0 & 1 & 1 & 0 & 1 & 2 \\
\hline $\mathbf{5}$ & 1 & 0 & 0 & 0 & 1 & 7 \\
\hline $\mathbf{6}$ & 0 & 1 & 4 & 0 & 2 & 10 \\
\hline $\mathbf{7}$ & 0 & 0 & 1 & 5 & 4 & 5 \\
\hline $\mathbf{8}$ & 0 & 0 & 2 & 2 & 1 & 10 \\
\hline 9 & 0 & 0 & 0 & 1 & 9 & 38 \\
\hline TOTAL & 2 & 2 & 8 & 8 & 18 & \multirow{2}{*}{1} \\
\hline
\end{tabular}


TABLA III. NIVELES DE PSA EN EL DIAGNÓSTICO Y EN EL SEGUIMIENTO.

\begin{tabular}{|l|c|c|c|c|c|}
\hline & $\begin{array}{c}\text { PSA } \\
\text { inicial }\end{array}$ & $\begin{array}{c}\text { PSA inicio } \\
\text { RT }\end{array}$ & $\begin{array}{c}\text { PSA } \\
\text { fin RT }\end{array}$ & $\begin{array}{c}\text { PSA a los } \\
\text { 2 meses }\end{array}$ & $\begin{array}{c}\text { PSA } \\
\text { nadir }\end{array}$ \\
\hline Media & 29,8 & 4,1 & 2,2 & 3 & 0,82 \\
\hline Mediana & 13 & 2,2 & 0,5 & 0,2 & 0,1 \\
\hline Mínimo & 2,6 & 0,00 & 0,00 & 0,00 & 0,00 \\
\hline Máximo & 475 & 19 & 19,5 & 84,0 & 8,8 \\
\hline
\end{tabular}

PSA: Antígeno Prostático Específico; RT: Radioterapia.

Toxicidad gastrointestinal. El $45 \%$ de los pacientes no comunicaron síntomas digestivos, el $35 \%$ describió alteraciones grado 1. La toxicidad de grado 2 se describió en el $14,3 \%$ de los pacientes y grado 3 sólo en un paciente.

\section{Hormonoterapia}

En el periodo de tiempo asistencial y cohorte de pacientes analizados no existió un criterio homogéneo en su indicación.

Veinticinco pacientes realizaron tratamiento hormonal concomitante o adyuvante a la RT. Estos pa- cientes presentaban Gleason $>7$ y/o PSA $>10 \mathrm{ng} / \mathrm{ml}$ en el momento de la recidiva. En los 10 pacientes que progresaron tras la finalización de la RT, la hormonoterapia (HT) se empleó como único tratamiento de rescate, habitualmente con administración de un antiandrógeno y un análogo de la LHRH.

\section{Seguimiento}

No existen pérdidas en el seguimiento. 10 pacientes progresaron tras la RT, 2 recidivas locales, 1 recidiva locorregional y 7 metástasis a distancia, (predominantemente óseas). En tres pacientes se diagnosticó un segundo tumor primario (2 cánceres

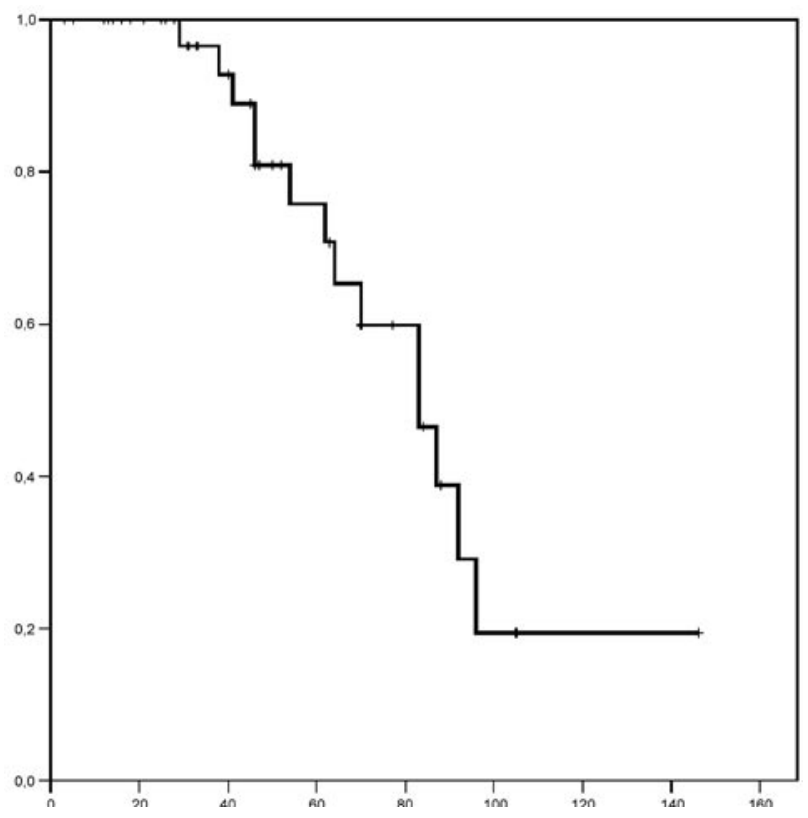

FIGURA 2. Supervivencia libre de fracaso bioquímica. bioquímica.

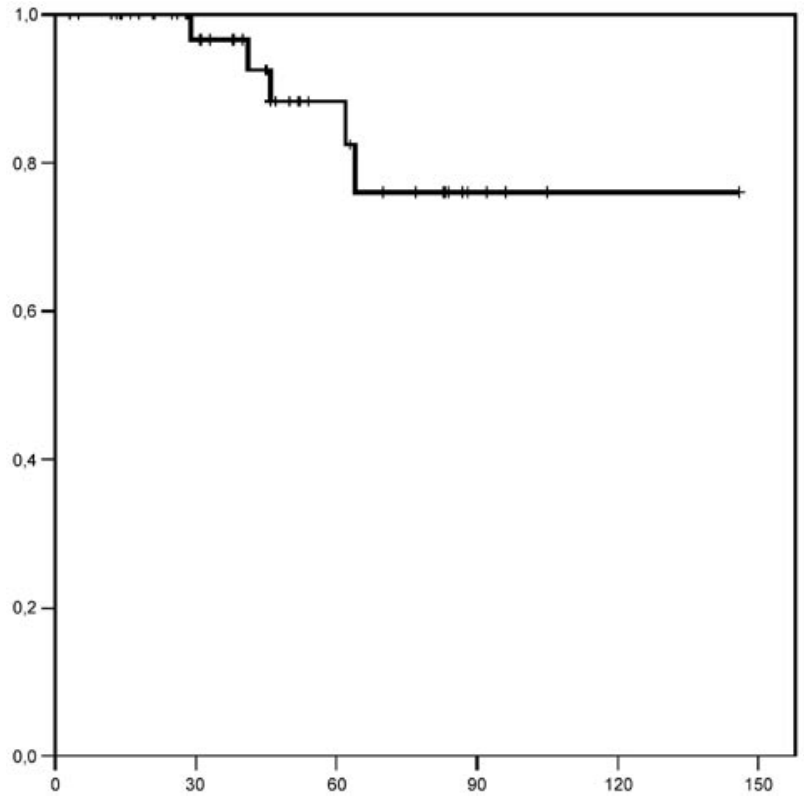

FIGURA 1. Supervivencia global de la serie. 


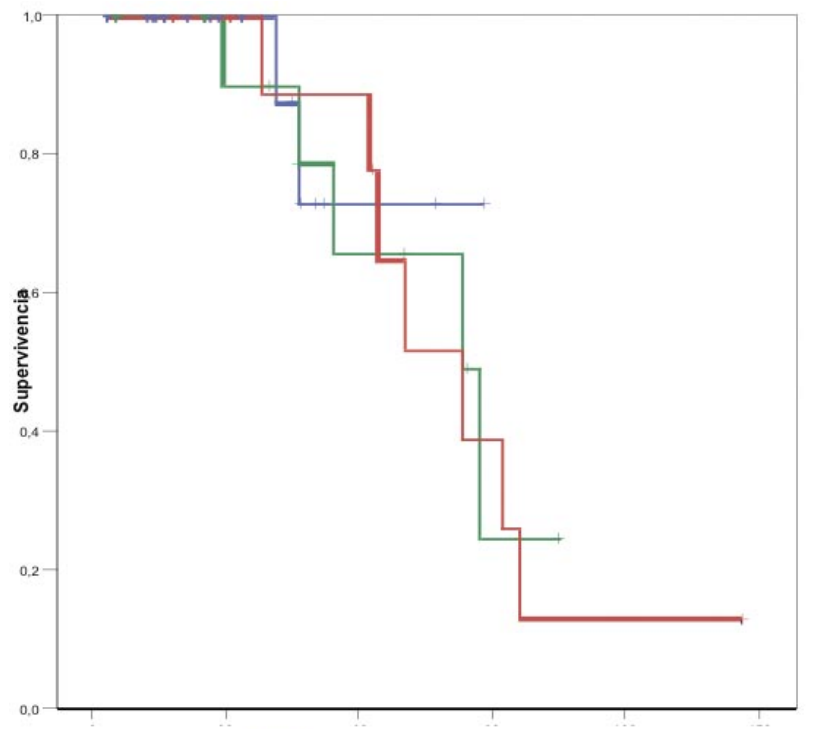

PSA: $0-2 \mathrm{ng} / \mathrm{ml}$ (azul)

PSA: $2,01-5 \mathrm{ng} / \mathrm{ml}$ (verde)

PSA: $5,01-20 \mathrm{ng} / \mathrm{ml}($ roja $)$

$\mathrm{p}=0,03$

FIGURA 3. Supervivencia libre de progresión bioquímica según los niveles de PSA al inicio de la radioterapia.

de pulmón y un sarcoma pélvico). 5 pacientes han fallecido (12\%) por cáncer de próstata. Con una mediana de seguimiento de 70 meses, la SG a los 5 años desde la recidiva fue de $86 \pm 6 \%$ (Figura 1). La SLF a los 5 años fue del $76 \pm 4 \%$ (Figura 2). La supervivencia causa específica fue del $93 \pm 5 \%$ a los 5 años. En el análisis univariante, los factores adversos para la recidiva encontrados en la serie fueron la persistencia de PSA elevado tras la cirugía $(p=0,05)$, las cifras de PSA $>2 \mathrm{ng} / \mathrm{ml}$ al inicio de la RT $(p=0,03)$ (Figura 3) y los niveles de Gleason en la cirugía entre 8 y $10(p=0,01)$. La presencia de borde afecto, en el presente análisis, no influyó en el riesgo de fracaso bioquímico después de la RT.

\section{DISCUSIÓN}

Los resultados presentados por grupos cooperativos internacionales $(13,14)$ en el último año comunican que la RT adyuvante tras prostatectomía es eficaz en determinados grupos de riesgo lafectación extracapsular, margen de resección afecto y/o afectación de las vesículas seminales), en términos de supervivencia libre de fracaso bioquímico. Estos datos contribuyen a definir el papel de la RT tras la prostatectomía que se consideraba controvertido en la literatura médica $(3,5,9,15,16)$.
Sin embargo, existen aspectos polémicos sobre sus indicaciones. Su utilización en todos los pacientes de riesgo, sin considerar factores como la edad, el grado de duplicación del PSA o la extensión del margen quirúrgico afecto, puede interpretarse como un sobretratamiento, máxime cuando la supervivencia global no se ve alterada por la RT adyuvante $(8,9,13)$. El precedente bibliográfico más numeroso se centra en investigar la RT como tratamiento de rescate, bien por persistencia de PSA elevado tras cirugía, bien por recidiva bioquímica en el curso del tratamiento $(4,16-18)$.

La prostatectomía radical modifica la estadificación clínica con migración de los casos hacia estadios de pronóstico más adverso, de modo que la mayor parte de los pacientes remitidos a RT por recidiva bioquímica o persistencia corresponden a estadios más avanzados o con incremento del índice del Gleason en más del $65 \%$ de los casos (6,7). A pesar de los avances de las técnicas de imagen en los últimos años, se ha comprobado que en un porcentaje próximo al $50 \%$ se descubre afectación microscópica en la cápsula prostática y/o en las vesículas seminales que no habían sido identificadas en la ecografía transrectal ni en la TAC (19). La información sobre la extensión del tumor (la T de la clasificación TNM) que aportan la TAC, la RMN y la ecografía transrectal tiene escaso valor predictivo sobre los hallazgos quirúrgicos, lo que explica que muchos pacientes en el presente análisis fueran operados a pesar de tratarse de estadios localmente avanzados.

El grado de Gleason ha sido insistentemente señalado en muchas series como uno de los factores de riesgo que más influyen en la recaída. Son numerosos los estudios en los que se ha demostrado que los pacientes con grados de Gleason iguales o superiores a 7 se asocian a un pronóstico desfavorable y se les considera candidatos a tratamiento adyuvante, tanto local como sistémico $(5-7,19)$. En nuestra serie, los pacientes con un Gleason $>7$ tras la cirugía recibieron $\mathrm{RT}+\mathrm{HT}$ en el momento de la progresión bioquímica.

La extirpación radical de la próstata resulta técnicamente compleja debido a su íntima relación con las estructuras anatómicas cercanas (20). La aparición de márgenes de resección afectos se interpreta, en la mayoría de los casos, como una extensión tumoral extracapsular a la grasa periprostática o al cuello vesical. La presencia de límites quirúrgicos afectos, además de estar relacionada con la $T$, como se demuestra en el presente análisis, se describe con mayor frecuencia en los tumores pobremente diferenciados $(6-8,19-21)$. Van den Ouden y cols. (23) demostraron que los márgenes quirúrgicos positivos eran un factor adverso independiente para la recidiva y con impacto 
sobre la supervivencia libre de fracaso bioquímico tras la prostatectomía. Sin embargo, es importante enfatizar que la presencia de márgenes positivos no es sinónimo de recidiva bioquímica (20-23).

En el estudio de Swindle y cols. (24), sólo el $40 \%$ de los pacientes con margen positivo recidivaron. En la actualidad se agrupa la afectación del límite quirúrgico como focal, extensa o masiva. El hallazgo de un margen positivo focal no parece ser criterio único para prescribir RT adyuvante y la presencia de borde afecto no se considera factor adverso para la recidiva bioquímica tras la RT (20-23). Este hecho se explica, en parte, por el efecto de control pélvico de la RT y por la asociación, en los pacientes sin margen afecto tratados con RT, de otros factores comprometedores del pronóstico (Gleason >7, T3a y T3b).

Diversos estudios retrospectivos han publicado la superioridad de las tasas de control local en pacientes pT3 o con borde afecto cuando se les administra RT adyuvante tras la cirugía $(4,9,15,21,25)$. Los trabajos de Petrovich y cols., con RT adyuvante y más de 400 pacientes analizados de forma retrospectiva, comunican un porcentaje de recurrencia menor del $10 \%$, con una SLFB del $69 \%$ y una SG del $92 \%$ a los 5 años $(4,26)$. Otros estudios realizados en los años 90 mostraron cifras similares, con una tasa de recidiva local en pT3-pT4 de un 30-40\% de los pacientes tratados con cirugía exclusiva frente a un $0-8 \%$ de los tratados con cirugía y RT adyuvante $(21,27,28)$.

Las experiencias clínicas con RT de rescate por recidiva o persistencia bioquímica han insistido en la ineficacia de la RT cuando se administra con niveles de PSA superiores a $2 \mathrm{ng} / \mathrm{ml}(15,16,29,30)$. En este subgrupo de pacientes se describen SLFB próximas al $30 \%$ frente al $75-80 \%$ en los pacientes con PSA $<2 \mathrm{ng} / \mathrm{ml}$ al inicio de la RT, dato confirmado en el presente estudio. Investigar si la administración de RT adyuvante en pacientes de riesgo es más beneficiosa que la RT de rescate, siempre que los niveles de PSA no superen los $2 \mathrm{ng} / \mathrm{ml}$, es una cuestión pendiente de abordar. La imposibilidad de hacer estudios aleatorizados que resuelvan la pregunta y la dificultad de comparar dos indicaciones distintas de la RT (adyuvante vs rescate) prescritas en grupos de pacientes distintos (pacientes sin recidiva y con recidiva bioquímica, respectivamente) dejan a los estudios prospectivos como la única arma metodológica capaz de aportar información convincente en este dilema.

Respecto a la técnica de RT tampoco existe consenso sobre la adecuación de los volúmenes y la dosis. Las recomendaciones de los estudios res- pecto al volumen son la irradiación del lecho prostático con márgenes de $1 \mathrm{~cm}$, establecidos en las imágenes obtenidas en la TAC previa a la cirugía. No se recomienda de forma sistemática la irradiación pélvica ganglionar, salvo en los pacientes con ganglios afectados histológicamente tras la prostatectomía o con recidiva pélvica evidente en la TAC $(4,29,31)$.

Dosis de 60-65 Gy, con un fraccionamiento convencional, son recomendadas en los estudios aleatorizados que exploran la RT adyuvante $(13,14)$. En las series retrospectivas, la dosis total ha oscilado entre 45 y $74 \mathrm{~Gy}$, apareciendo como única recomendación la de administrar dosis superiores a 61 Gy para mejorar la SLFB $(4,5,9,16-18,29,30)$. En nuestra serie, el lecho de prostatectomía fue el volumen más empleado con una dosis media de 66 Gy. La dificultad técnica para su delimitación también se ha señalado por múltiples autores, describiéndose que, en general, su volumen es incluso superior al volumen prostático empleado en los pacientes tratados con RT de intensidad modulada (IMRT) (32).

La toxicidad que se induce como consecuencia del tratamiento de RT suele ser escasa, con desarrollo de patología severa de manera muy excepcional. Se recomienda no iniciar la RT hasta la recuperación de la incontinencia urinaria postquirúrgica, lo que representa un tiempo medio de espera de 3 meses en la RT adyuvante $(32,33)$.

La cistitis y la rectitis clínicamente significativas se registran en un porcentaje aproximado del $5 \%$ al $10 \%$ de los pacientes $y$, en nuestra casuística, es habitual conseguir su control mediante tratamientos médicos conservadores. Zelefsky y cols. (34) describieron que, tras la administración de una dosis media de 64,8 Gy, el 7\% de los pacientes referían toxicidad genitourinaria grado 2 y el $21 \%$ toxicidad gastrointestinal grado 2. No observaron casos de toxicidad grado 3. En los pacientes que tenían urgencia miccional, ésta empeoró en los 6 meses siguientes al tratamiento de RT y se resolvió aproximadamente a los 12 meses de finalizarla. La urgencia miccional aumenta su frecuencia en aquellos pacientes sometidos a RT y cirugía (12-15\%) respecto a los quirúrgicos exclusivos (5-10\%), aunque estas diferencias no son significativas al analizar los resultados a los 6 meses $(35,36)$.

El momento en el que se administra la RT así como su indicación parece influir en la incidencia e intensidad de la toxicidad, aumentando en aquellos casos en los que se utiliza como tratamiento de rescate, posiblemente por la utilización de dosis acumulativas mayores $(25,29,30)$. 
Por último, la diversidad e inconsistencia en la indicación de la HT, en el presente análisis, no permite concluir sobre su contribución tras la prostatectomía. En la actualidad, están activos dos estudios aleatorizados (RTOG 9601 y RTOG p0011) que comparan la eficacia de la RT adyuvante frente a la $\mathrm{RT}+\mathrm{HT}$ adyuvantes en los pacientes de alto riesgo. Sin embargo, la cancelación del brazo con HT como único tratamiento de rescate, nos impedirá saber cuál es su verdadera eficacia en estos pacientes.

Con las limitaciones inherentes del presente análisis (tamaño muestral y carácter retrospectivo), los datos observados destacan el interés de indicar la RT de rescate de forma precoz y la necesidad de mejorar la estadificación antes del tratamiento inicial. La selección individualizada y consensuada de los pacientes que se pueden beneficiar de la RT adyuvante centra la discusión actual en los comités de evaluación oncológica interdisciplinarios. La publicación de los estudios de la EORTC (13) y del SWOG (14) han impulsado un profundo cambio conceptual $y$ asistencial, con un aumento en el número de pacientes tratados en nuestra institución con RT adyuvante (10 pacientes en los últimos 6 meses). Su seguimiento a largo plazo y la comparación con los pacientes de esta cohorte histórica permitirá afinar el conocimiento sobre las indicaciones de la RT tras prostatectomía y sobre aspectos de optimización técnica de la irradiación.

\section{BIBLIOGRAFÍA y LECTURAS RECOMENDADAS ( ${ }^{*}$ lectura de interés $y^{* *}$ lectura fundamental)}

1. JEMAD, A.; SIEGEL, R.; WARD, E. y cols.: "Cancer Statistics, 2006". CA Cancer J. Clin., 56: 106, 2006.

2. INE.: "Causas de muerte en España". 2000.

3. VAN POPPEL, H.; VANUYTSEL, L.; PETRO$\mathrm{VICH}$, Z. y cols.: "Is postoperative irradiation after radical prostatectomy necessary?". Eur. J. Cancer, 35: 1763, 1999.

4. PETROVICH, Z.; LIESKOVSKY, G.; LANGHOLZ, B. y cols.: "Postoperative radiotherapy in 423 patients with pT3N0 prostate cancer". Int. J. Radiat. Oncol. Biol. Phys., 53: 600, 2002.

*5. VALICENTI, R.K.; GOMILLA, L.G.; PEREZ, C.A.: "Radiation therapy after radical prostatectomy: a review of the issues and options". Sem. Radiat. Oncol., 13: 130, 2003.

6. BABAIAN, R.J.; TRONCOSO, P.; BHADKAMKAR, V.A. y cols.: "Analysis of clinicopathologic factors predicting outcome after radical prostatectomy". Cancer, 91: 1414, 2001.
*7. KRÜGER, B.; SCHRÖDER, F.H.: "Risk factors for progression in patients with prostate cancer treated by radical prostatectomy". Curr. Opin. Urol., 7: 186, 1997.

8. AKDUMAN, B.; CRAWFORD, E.D.: "The management of high risk prostate cancer". J. Urol., 169: 1993, 2003.

*9. DO, L.V.; DO, T.M.; SMITH, R. y cols.: "Postoperative radiotherapy for carcinoma of the prostate: impact on both local control and distant diseasefree survival". Am. J. Clin. Oncol., 25: 1, 2002.

10. HAUKAAS, S.A.; HALVORSEN, O.J.; DAEHLIN, L. y cols.: "Is preoperative serum prostate-specific antigen level significantly related to clinical recurrence after radical retropubic prostatectomy for localized prostate cancer?". BJU Int., 97: 51, 2006.

11. COX, J.D.; STETZ, J.; PAJAK, T.F.: “Toxicity criteria of the Radiation Therapy Oncology Group (RTOG) and the European Organization for Research and Treatment of Cancer (EORTC)". Int. J. Radiat. Oncol. Biol. Phys., 31: 1341, 1995.

12. ICRU: "Prescribing, recording and reporting Photon Beam Therapy (Supplement to ICRU Report 50)". ICRU report 62, November 1999.

**13. BOLLA, M.; VAN POPPEL, H.; COLLETTE, L. y cols.: "Postoperative radiotherapy after radical prostatectomy: a randomised controlled trial (EORTC trial 22911)". Lancet, 366: 572, 2005.

**14. SWANSON, G.P.; THOMPSON, I.M.; TANGEN, C. y cols.: "Phase III randomized study of adjuvant radiation therapy versus observation in patients with pathologic T3 prostate cancer (SWOG 8794)”. Int. J. Radiat. Oncol. Biol. Phys., 63: 1, 2005.

15. HENNEQUIN, C.; RAVERY, V.; MAYLIN, C. y cols.: "Post-prostatectomy radiotherapy: for which patients and when?". Cancer Radiother., 6: 168, 2002.

*16. CATTON, C.; GOSPODAROWICZ, M.; WARDE, P. y cols.: "Adjuvant and salvage radiation therapy after radical prostatectomy for adenocarcinoma of the prostate". Radiother. Oncol., 59: 51, 2001.

*17. BOTTKE, D.; WIEGEL, T.; HOCHT, S. y cols.: "Salvage radiotherapy in patients with persisting prostate-specific antigen after radical prostatectomy for prostate cancer". Oncology, 65: 18, 2003.

18. PEYROMAURE, M.; ALlOUCH, M.; ESCHWEGE, F. y cols.: "Salvage radiotherapy for biochemical recurrence after radical prostatectomy: a study of 62 patients". Urology, 62: 503, 2003.

19. BLUTE, M.L.; BERGSTRALH, E.J.; IOCCA, A. y cols.: "Use of Gleason score, prostate specific antigen, seminal vesicle and margin status to 
predict biochemical failure after radical prostatectomy". J. Urol., 165: 119, 2001.

20. JONES, E.: "Resection margin status in radical retropubic prostatectomy specimens: relationships to type of operation, tumor size, tumor grade and local tumor extension". J. Urol., 44: 89, 1990.

*21. CHOO, R.; HRUBY, G.; HONG, J. y cols.: "Positive resection margin and/or pathologic $\mathrm{T} 3$ adenocarcinoma of prostate with undetectable postoperative PSA after radical prostatectomy: to irradiate or not?". Int. J. Radiat. Oncol. Biol. Phys., 52: 674, 2002.

*22. GROSSFELD, G.D.; TIGRANI, V.S.; NUDELL, D. y cols.: "Management of a positive surgical margin after radical prostatectomy; decision analysis". J. Urol., 164: 93, 2000.

23. VAN DEN OUDEN, D.; SCHRODER, F.H.: "The treatment of locally advanced T3 prostatic carcinoma using radical prostatectomy or radiotherapy. A review". Tijdschr. Gerontol. Geriatr., 29: 74, 1998.

24. SWINDLE, P.; EASTHAM, J.A.; OHORI, M. y cols.: "Do margins matter? The prognostic significance of positive surgical margins in radical prostatectomy specimens". J. Urol., 174: 903, 2005.

$* * 25$. ANSCHER, M.S.: "Adjuvant radiotherapy following radical prostatectomy is more effective and less toxic than salvage radiotherapy for a rising prostate specific antigen". Int. J. Cancer, 96: 91, 2001.

*26. PETROVICH, Z.; LIESKOVSKY, G.; STEIN, J.P. y cols.: "Comparison of surgery alone with surgery and adjuvant radiotherapy for pT3N0 prostate cancer". BJU Int., 89: 604, 2002.

27. SCHAFER, U.; WITT, F.; MICKE, O. y cols.: "Adjuvant radiotherapy in locally confined prostate cancer". Anticancer Res., 23: 983, 2003.

28. LEIBOVICH, B.C.; ENGEN, D.E.; PATTERSON, D.E. y cols.: "Benefit of adjuvant radiation therapy for localized prostate cancer with a positive surgical margin”. J. Urol., 16: 1178, 2000 .
*29. CHOO, R.; HRUBY, G.; HONG, J. y cols.: “(In)Efficacy of salvage radiotherapy for rising PSA or clinically isolated local recurrence after radical prostatectomy". Int. J. Rad. Oncol. Biol. Phys., 53: 269, 2002.

30. HOCHT, S.; WIEGEL, T.; SCHOSTAK, M. y cols.: "Adjuvant and salvage radiotherapy after radical prostatectomy". Onkologie, 5: 201, 2002.

31. HOCHT, S.; WIEGEL, T.; BOTTKE, D. y cols.: "Computed tomogram prior to prostatectomy. Advantage in defining planning target volumes for postoperative adjuvant radiotherapy in patients with stage C prostate cancer?'. Strahlenther Onkol., 178: 134, 2002.

32. THE, B.S.; MAI, W.Y.; AUGSPURGER, M.E. y cols.: "Intensity modulated radiation therapy (IMRT) following prostatectomy: more favourable acute genitourinary toxicity profile compared to primary IMRT for prostate cancer". Int. J. Radiat. Oncol. Biol. Phys., 49: 465, 2001.

*33. FORMENTI, S.C.; LIESKOVSKY, G.; SKINNER, D. y cols.: "Update on impact of moderate dose of adjuvant radiation on urinary continence and sexual potency in prostate cancer patients treated with nerve-sparing prostatectomy". Urology, 56: 453, 2000.

*34. ZELEFSKY, M.J.; ASCHKENASY, E.; KELSEN, S. y cols.: "Tolerate and early outcome results of postprostatectomy three-dimensional conformal radiotherapy". Int. J. Radiat. Oncol. Biol. Phys., 39: 37, 1997.

**35. VAN CANGH, P.J.; RICHARD, F.; LORGE, F. y cols.: "Adjuvant radiation therapy does not cause urinary incontinence after radical prostatectomy: results of a prospective randomized study". J. Urol., 159: 164, 1998.

*36. HOFMANN, T.; GAENSHEIMER, S.; BUCHNER, A. y cols.: "An unrandomised prospective comparison of urinary continence, bowel symptoms and the need for further procedures in patients with and with no adjuvant radiation after radical prostatectomy”. BJU Int., 92: 360, 2003. 